\title{
SURFACE INVESTIGATION BY SCANNING TUNNELING MICROSCOPY IN LIQUID MEDIUM
}

\author{
M. Phaner ${ }^{a}$, Y. Robach ${ }^{a}$, C. DE Villeneuve ${ }^{a}$, S. WojczuK ${ }^{b}$, \\ W. OlejniczaK ${ }^{b}$ AND L. Porte ${ }^{a}$ \\ a Département de Physicochimie des Matériaux, Ecole Centrale de Lyon \\ BP 163, 69131 Ecully, France \\ ${ }^{b}$ Department of Solid State Pliysics, University of Lódź \\ Pomorska 149/153, 90-236 Eódz, Poland
}

Scanning tunneling microscopy in liquid environment has gained an increased interest in recent years. The specific features of the in silu observation of surface structures at the solid/liquid interface are first presented. Next, a high resolution imaging of high oriented pyrolytic graphite (HOPG) graphite surface shows the potentiality of our home-made microscope. The last study, performed on technological InP substrates, illustrates the extensive applications of in situ imaging in the fields of semiconductor technology.

PACS numbers: $61.16 . \mathrm{Ch}, \mathbf{7 3 . 4 0 . G k}$

\section{Introduction}

Scanning tunneling microscopy (STM) has become a powerful tool for topographic imaging and analysis of conducting and semiconducting surfaces with atomic resolution, in various environments. As STM in air is mainly restricted to graphite and noble metals investigations, most applications have concerned themselves with the study of clean or ideal surfaces in ultrahigh vacuum. Nevertheless, since the pionecring work of Hansma's group concerned with the study of surfaces immersed in liquid solutions [1-3], considerable attention has recently been focused on in situ observations of surface structures at the solid/liquid interface, under potentiostatic control [4-8], with - in some cases - atomic resolution. Advantages of the solid/liquid interface are such that the liquid can replace an unknown and inhomogeneous layer of contaminants or it can protect against oxidation air sensitive materials like semiconductors: high-resolution imaging of technical semiconductor surfaces ( $\mathrm{Si}, \mathrm{GaAs})$ [9-12] has been successfully performed in acidic $\left(\mathrm{H}_{2} \mathrm{SO}_{4}\right)$ or basic $(\mathrm{NaOH})$ medium under potentiostatic control. On the other hand, solid/liquid interface studies offer extensive possibilities for in situ STM observation of electrode surface modifications, in the field of electrochemical processes as it was first pointed out by Arvia [13]. As examples, metal electrodeposition [14-17] on metals 
or semiconductors, photocorrosion of semiconductors $[11,14]$ have already becn studied by several groups.

As the presence of a liquid medium imposes some specific characteristics and experimental restrictions, we will first describe, in this paper, the principal aspects of in situ STM in electrolytic systems, by restricting our discussion to the case of conducting liquids. After a brief description of our home-made microscope, we next illustrate our purpose with results obtained on graphite and indium phosphide (InP) surfaces respectively.

\section{In silu scanning tunneling microscopy features}

In situ scanning tunneling microscopy is quite singular. The presence of liquid between tip and sample leads, when a potential difference is applied, to the creation of an electrochemical cell. Thus, electron transfers can occur at the tip/electrolyte and sample/electrolyte interfaces. These reactions can affect drastically the STM experiments. First, a faradaic current appears which may disguise the much smaller tunneling current. Then the surfaces of both tip and sample can be modified by some deposition or corrosion processes. A linkage between electrochemical processes at the substrate and at the laterally exposed parts of the tip is also possible. The charge transport between the two interfaces is performed by the electrochemical reactants.

- It is obvious that these electrochemical processes must be prevented as far as possible to achieve STM observations in liquids. Charge transfers between laterally exposed parts of the tip and substrate can be reduced by using "insulated tips" except for the very end. The conducting extremity provides tunneling transfer. Electrochemical processes on substrate and tip can be controlled by a "potentiostatic system", coupled with the STM unit, which allows to minimize the faradaic current. However, the last problem concerns the possible influence of the tunneling current on the electrochemical processes.

The potentiostatic system is used to control the electrochemical reactions on the sample and to limit those which can arise on the tip. It consists of an electrochemical cell with a three electrodes configuration coupled with a potentiostat. A schematic representation is shown in Fig. 1a. The potentiostatic circuit is separated into two parts. The first one is a feedback loop which adjust the potential of the sample - the working electrode (WE) - versus a reference electrode (RE). The second one is an electrolytic circuit obtained by applying a potential difference between the working electrode and a counter electrode (CE). A faradaic current can flow in this circuit. In this three electrodes configuration, no current can pass through the reference electrode. Its potential remains at the same value allowing the working electrode potential $E_{\mathrm{W} / \mathrm{R}}$ to be defined with precision. The behavior of the faradaic current versus this potential is determined by current-potential curves (Fig. 1b). Therefore, the potentiostat enables to define the sample potential (versus reference electrode) $E_{\mathrm{W} / \mathrm{R}}$ and then the faradaic current $I_{\mathrm{W} / \mathrm{C}}$. Added to that, in an in silu STM experiment, it can also control the tip potential $E_{\mathrm{T} / \mathrm{R}}$. The tip appears like a fourth electrode held at a constant bias voltage $V_{\mathrm{t}}$ relative 
(a)

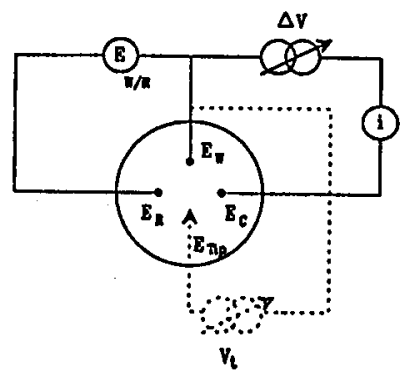

(b)

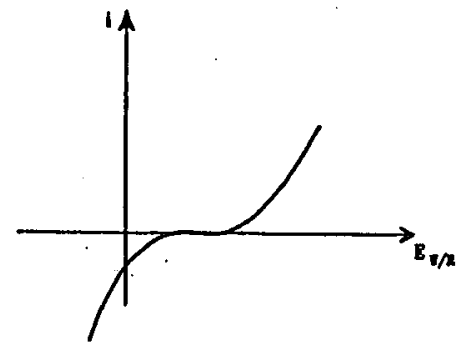

Fig. 1. (a) Schematic representation of the electrolytic cell and the potentiostatic circuit in a three electrodes configuration. The STM tip-sample circuit is represented in dashed line. (b) Schematic current-voltage curve.

to the working electrode

$$
V_{\mathrm{t}}= \pm\left(E_{\mathrm{W} / \mathrm{R}}-E_{\mathrm{T} / \mathrm{R}}\right)
$$

For a defined potential $E_{\mathrm{W} / \mathrm{R}}$, it is possible to adjust the potential $E_{\mathrm{T} / \mathrm{R}}$ at a value corresponding to a low faradaic current between tip and counter electrode $I_{\mathrm{T} / \mathrm{C}}$. In that case, the tip plays the role of another working electrode. However, it can be considered also as a counter electrode, because an electrochemical current can appear between tip and sample when tunneling voltage is applied. One can get rid of this embarrassing current by a judicious choice of the tunneling voltage.

In general, the potentiostatic control is used to minimize both faradaic currents $I_{\mathrm{T} / \mathrm{C}}$ and $I_{\mathrm{W} / \mathrm{C}}$ in order to allow STM observations of the sample surface without modifications. Nevertheless, it can also enable to create reactions on the sample surface such as deposition or corrosion $\left(I_{\mathrm{W} / \mathrm{C}} \neq 0\right)$ which can be seen in real time by in situ STM. Electrochemists are of course really interested in this other possibility.

Insulated tips are prepared from conventional tips by covering with different insulators such as glass capillaries $[1,18,19]$, fingernail polish [20], epoxy coverage $[21,22]$, varnishes [23] and apiezon wax coverage [5, 24]. The last one is often employed, because the grease is easy to use and is found to be inert in most electrolytes. 


\section{Experimental design}

The electrochemical cell of our home-made microscope consists of a small cylindric plexiglass beaker. Within the cell, three electrodes are situated: the sample under study representing the working electrode is situated in the center of the cell, and surrounded by a Pt ring counter electrode, at the bottom of the cell. In close proximity to the sample, a micro $\mathrm{Ag} / \mathrm{AgCl}$ electrode is used as a reference electrode; it is confined in contact with a $1 \mathrm{M} \mathrm{NaCl}$ solution in a small separate compartment which is connected to the main part of the cell by a $\mathrm{ZrO}_{2}$ frit. The sample is attached with silver paint to a back grounded contact and tunneling bias is applied to the tip. Sample edges are covered with a resin to ensure an accurate isolation. In this way, the electrolyte is only in contact with chemically inert material.

Our tips were prepared from $0.2 \mathrm{~mm} 90 \% \mathrm{Pt}-10 \%$ Ir wires which were subsequently electrochemically sharpened in cyanide solutions $(6 \mathrm{M} \mathrm{NaCN}+2 \mathrm{M} \mathrm{KOH})$ and covered with a thin insulating layer of apiezon wax except for the frontmost part. Our insulated tips lead to leakage currents of less than $50 \mathrm{pA}$.

The tip is bound to a cylindrical shaped piezotube following the design of Binnig and Smith [25]. Approach of the tip is made using a controlled step-by-step motor. Image recording is computer processed and STM measurements performed in the slow scan constant current mode $(2 \mathrm{~Hz} /$ line $)$.

In the following part, we present two examples of in situ investigation under potentiostatic control of namely graphite and InP surfaces.

\section{Examples of surface imaging under potentiostatic control}

\subsection{In situ high resolution images of graphite}

A high-resolution experiment on graphite was done to evaluate the performance of the apparatus. The image reported in Fig. 2 presents graphite atoms on a $26 \AA \times 26 \AA$ scanning area. Atomic rows are separated by $2.46 \AA$ which corresponds to the unit cell that can be observed by STM. This image was carried out in a $1 \mathrm{M} \mathrm{NaCl}$ solution, with a tunneling current $I_{\mathrm{t}}=-5.6 \mathrm{nA}$, a bias voltage $V_{\mathrm{t}}=130 \mathrm{mV}$ (tip polarized positive with respect to the sample) and a sample potential versus $\mathrm{Ag} / \mathrm{AgCl}, E_{\mathrm{W} / \mathrm{R}}=-200 \mathrm{mV}$. We found atomic corrugations within 1-2 $\AA$. The quality of this image presented without image enhancement confirms the ability to obtain atomic resolution images in liquid environments.

\subsection{In situ InP surface images in acidic solulion}

An evaluation of the surface topography of technological InP substrates is of great interest, since they represent the starting material for most device processing. They have potential interest for both optoelectronic and microwave device applications. In particular, high-quality InP surfaces must be obtained prior to epitaxial growth, since this step critically affects the overall properties of epitaxial layers of InP and its ternary and quaternary related compounds. 


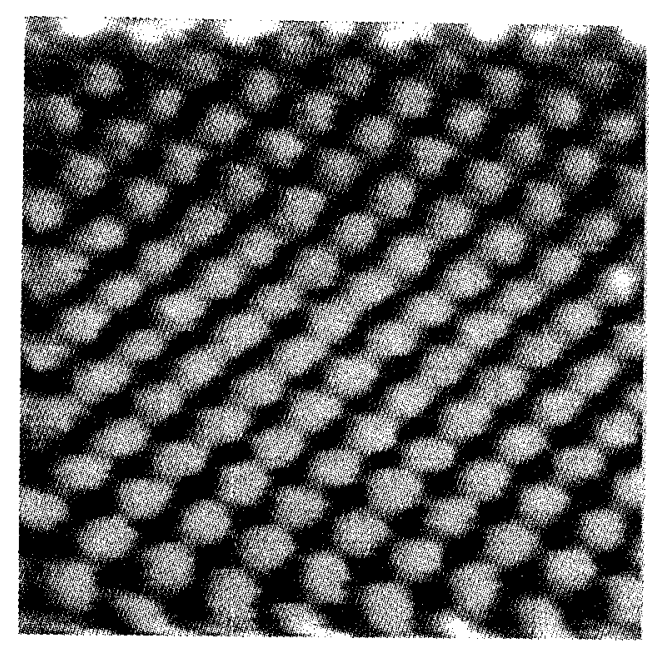

Fig. 2. High-resolution STM image ( $26 \AA \times 26 \AA$ ) of HOPG graphite obtained in $1 \mathrm{M}$ $\mathrm{NaCl}$.

The samples used were $n$-type (Sn doped) $\operatorname{InP}(100)$ wafers whose doping levels were in the range $3-4 \times 10^{18} \mathrm{~cm}^{-3}$. Two types of wafer were investigated. The first one (wafer $A$ ) was chemomechanically polished. The second one (wafer $B$ ) is an "epi-ready" substrate with specific surface preparation. Before STM investigations, the wafer surfaces were degreased in organic solvants, rinsed in deionized water and etched in 5\% HF solution to get rid of the native oxide. An ohmic contact was made by laying an In-Ga eutectic on the back side of the InP samples. Then, they were transferred into the STM cell and immediately covered by $0.25 \mathrm{M} \mathrm{H}_{2} \mathrm{NO}_{4}$ solution. The electrolyte concentration is high enough to protect the surface against oxidation but presents no risk for the electrochemical cell and the microscope. In order to determine the potential range allowing STM measurements we first studied the electrochemical behavior of tip and sample in the $\mathrm{H}_{2} \mathrm{SO}_{4}$ solution by recording current-potential curves in the dark. These voltamograms, reported in Fig. 3, allow to define potential regions where no electrochemical reactions occur and corresponding to the lowest faradaic currents $I_{\mathrm{W} / \mathrm{C}}$ and $I_{\mathrm{T} / \mathrm{C}}$. For the sample, the $E_{\mathrm{W} / \mathrm{R}}$ range is located between $-0.4 \mathrm{~V}$ and $+0.2 \mathrm{~V}$ versus $\mathrm{Ag} / \mathrm{AgCl}$ and for the tip $E_{\mathrm{TIR}}$ should be chosen between $+0.2 \mathrm{~V}$ and $+1 \mathrm{~V}$ versus $\mathrm{Ag} / \mathrm{AgCl}$. The relatively high doping level ensured a small depletion layer and consequently a low band bending (tunneling transfer can occur via interface states) as shown in Fig. 4a. Nevertheless, as the band bending is controlled by the semiconductor/electrolyte potential, we typically used a negative value of the sample potential $E_{\mathrm{W} / \mathrm{R}}(\approx-0.2 \mathrm{~V})$ to approach the accumulation regime (Fig. $\left.4 \mathrm{~b}\right)$ and to facilitate the tunneling transfer. Therefore, according to the possible values of $E_{\mathrm{T} / \mathrm{R}}$, the applied tunnel voltage was between $+0.4 \mathrm{~V}$ and $+1.2 \mathrm{~V}$, with positive bias to the tip.

We first investigated the surface topography of wafer $A$, corresponding to 

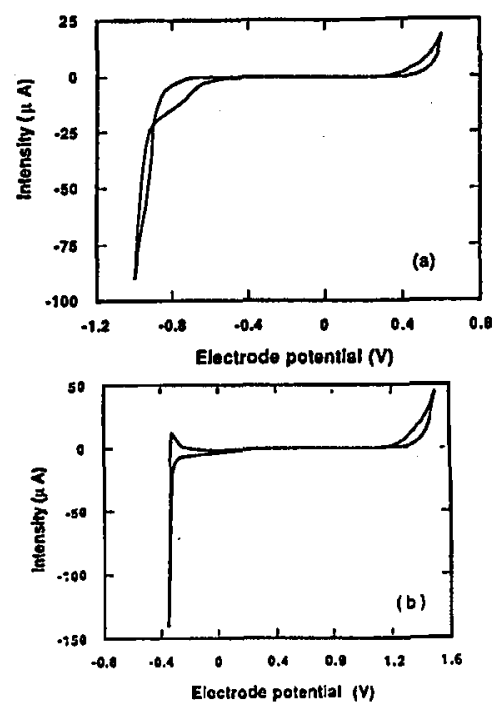

Fig. 3. Cyclic voltamograms of the InP (100) sample (a) and the Pt/Ir tip (b) obtained in the dark in $0.25 \mathrm{M} \mathrm{H}_{2} \mathrm{SO}_{4}$.

a)

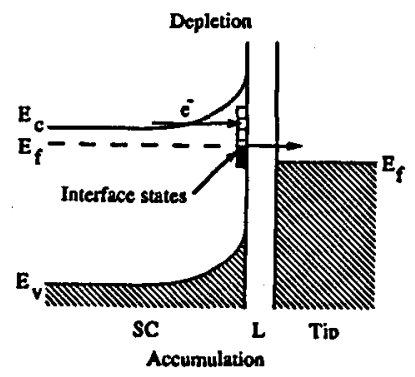

b)

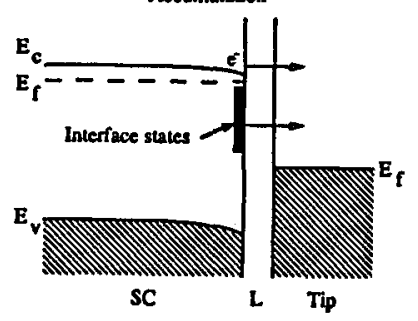

Fig. 4. Interfacial energetics for $n$-type semiconductor (SC)/liquid (L)/tip junction. 


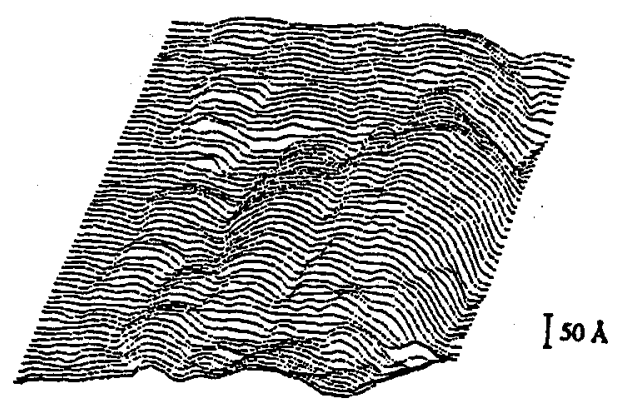

Fig. 5. STM image $(1700 \AA \times 1700 \AA)$ of the surface of a chemomechanically polished InP (100) substrate (wafer $A$ ) obtained in $0.25 \mathrm{M} \mathrm{H}_{2} \mathrm{SO}_{4}$.

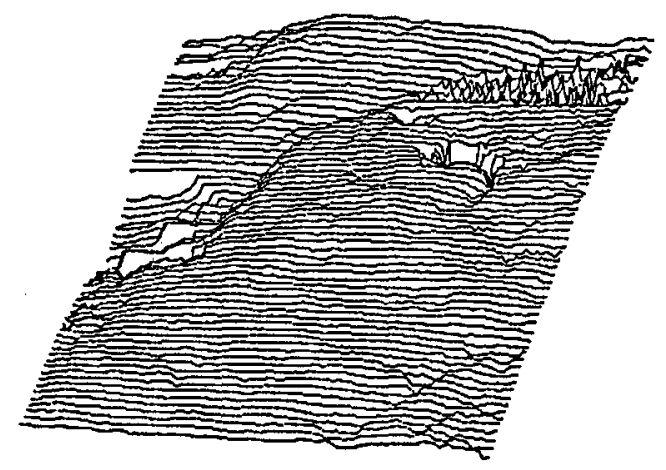

Fig. 6. STM image $(210 \AA \times 210 \AA)$ of the surface of an InP substrate presenting a small oxidized area.

a technological InP substrate chemomecanically polished. Figure 5 shows a large scale STM image $(1700 \AA \times 1700 \AA)$ obtained in $0.25 \mathrm{M} \mathrm{H}_{2} \mathrm{SO}_{4}$, which is representative of a large number of measurements performed at different points of the sample: it shows a moderately rough overall topography, characterized by a succession of "hillocks"; on this image, the maximum height difference is $\approx 200 \AA$, indicating that the hillocks are $\approx 50 \AA$ in height. This first result clearly shows the feasibility of in situ STM imaging of InP surfaces in aqueous sulfuric acid solution, which acts as an effective protective layer against oxidation. Nevertheless, we got on some images oscillations associated with the residual presence of small oxidized areas, as shown in Fig. 6. Stable and reproducible images were obtained over an extended time of $\approx$ two days. The surfaces remain stable in the low $\mathrm{pH}$ solution, at least long enough to ensure that the topographical features which we observe are representative of the surfaces in their initial conditions. The relatively high observed surface roughness did not allow us to obtain atomic resolution.

Comparison of images recorded on InP wafers $A$ and $B$ respectively must yield to a qualitative information on the surface quality consecutive to different 


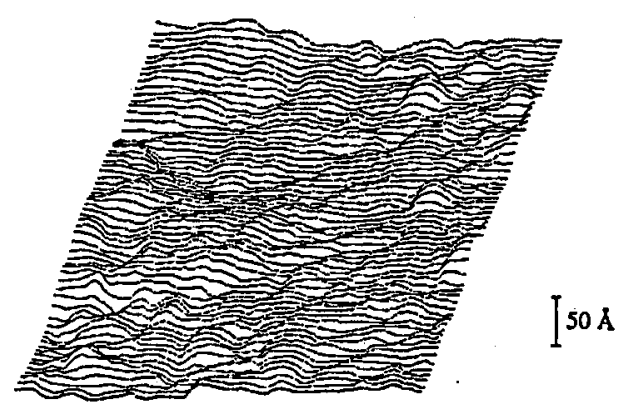

Fig. 7. STM image $(1700 \AA \times 1700 \AA)$ of the surface of an "epi-ready" InP (100) substrate (wafer $B$ ) obtained in $0.25 \mathrm{M} \mathrm{H}_{2} \mathrm{SO}_{4}$.

preparation procedures. Wafer $B$ corresponding to an "epi-ready" InP substratc, might be representative of the best surface preparation currently obtainable. As shown in Fig. 7 , a large scale image $(1700 \AA \times 1700 \AA)$ representative of wafer $B$, still shows a noticeable roughness, with small successive holes and islands. If we compare with Fig. 5, we may just notice a decreased size of the relief features, corresponding only to a slight improvement of the InP surface topography.

In comparison to the flat topography observed on technological Si substrates $[9,10,26]$, a noticeable improvement of InP surface preparation is still desirable. However, our results are comparable to that already observed on GaAs substrates [11].

\section{Conclusion}

This paper first outlined the specific features of STM imaging in conducting liquid environment. We concentrated our discussion on the necessity of a potentiostatic control and on the elaboration of isolated tips.

In situ observation of surfaces in aqueous solutions under potentiostatic control were performed using a home-made scanning tunneling microscope. The first study, relative to HOPG graphite, clearly shows that high-resolution imaging is attainable with such a microscope. In the second study, we checked and compared at a nanometer scale the surface quality of different InP technological substrates. This second experiment, performed on protected semiconductor surfaces, illustrates the wide possibilities which are offered by in situ STM imaging, in the field of semiconductor surface quality control. The feasibility of InP imaging in liquid environment would also be of practical interest in regard to the potential study of wet etching and cleaning procedures, or in the field of electrochemical applications.

\section{References}

[1] R. Sonnenfeld, P.K. Hansma, Science 232, 211 (1986).

[2] R. Sonnenfeld, B.C. Schardt, Appl. Phys. Lett. 49, 1172 (1986). 
[3] R. Sonnenfeld, J. Schneir, B. Drake, P.K. Hansma, D.E. Aspnes, Appl. Phys. Lelt. 50, 1742 (1987).

[4] A.A. Gewirth, A.J. Bard, J. Phys. Chem. 92, 5563 (1988).

[5] J. Wiechers, T. Twomey, D.M. Kolb, R.J. Behm, J. Electroanal. Chem. 248, 451 (1988).

[6] R. Christoph, H. Siegenthaler, H. Rolırer, H. Wiese, Eleclrochim. Acta 34, 1011 (1989).

[7] K. Uosaki, H. Kita, J. Vac. Sci. Technol. A 8, 520 (1990).

[8] K. Itaya, S. Sugawara, K. Sashikata, N. Furuya, J. Vac. Sci. Technol. A 8, 515 (1990).

[9] E. Tomita, N. Matsuda, K. Itaya, J. Vac. Sci. Technol. A 8, 534 (1990).

[10] R. Houbertz, U. Memmert, R.J. Behm, Appl. Phys. Lelt. 58, 1027 (1991).

[11] S. Eriksson, P. Carlsson, B. Holınström, K. Uosaki, J. Eleclroanal. Chem. 313, 121 (1991).

[12] K. Itaya, S. Sugawara, Y. Morita, II. Tokumoto, Appl. Phys. Lelt. 60, 2534 (1992).

[13] A.J. Arvia, Surf. Sci. 181, 78 (1987).

[14] T. Thundat, L.A. Nagahara, S.M. Lindsay, J. Vac. Sci. Technol. A 8, 539 (1990).

[15] R.S. Robinson, J. Vac. Sci. Technol. A 8, 511 (1990).

[16] K. Endo, S. Sugawara, S. Mishima, T. Okada, S. Morita, Jpn. J. Appl. Phys. 30, 2592 (1991).

[17] J. Schneir, V. Elings, P.K. Hansma, J. Electrochem. Soc. 135, 2774 (1988).

[18] H. Liu, F.R. Fan, C.W. Lin, A.J. Bard, J. Am. Chem. Soc. 108, 3838 (1986).

[19] K. Itaya, E. Tomita, Surf. Sci. 201, 507 (1988).

[20] M.P. Green, K. Hanson, D.A. Scherson, X. Xing, M. Richter, P.N. Ross, R. Carr, I. Lindau, J. Phys. Chem. 93, 2181 (1989).

[21] K. Uosaki, H. Kita, J. Electroanal. Chem. 259, 301 (1989).

[22] J. Schneir, V. Elings, P.K. Hansma, J. Electrochem. Soc. 135, 2774 (1988).

[23] A.A. Gewirth, D.H. Craston, A.J. Bard, J. Electroanal. Chem. 261, 477 (1989).

[24] L.A. Nagahara, T. Thundat, S.M. Lindsay, Rev. Sci. Instrum. 60, 3138 (1989).

[25] G. Binnig, D.P.E. Smith, Rev. Sci. Instrum. 57, 1688 (1986).

[26] E. Hartmann, P.O. Hahn, R.J. Behm, J. Appl. Phys. 69, 4273 (1991). 